\title{
Acute Kidney Injury Following Conversion From Laparoscopic to Open Cholecystectomy
}

\author{
Ahmed Dirweesh $^{\mathrm{a}, \mathrm{b}}$, Ritika Zijoo ${ }^{\mathrm{a}}$, Praneet Iyer ${ }^{\mathrm{a}}$
}

\begin{abstract}
Pneumoperitoneum during laparoscopic surgery is a complex physiologic event associated with neuroendocrine, respiratory, cardiovascular and renal disturbances as well as compromised organ blood flow. Impaired renal perfusion from pneumoperitoneum is attributed to intra-abdominal hypertension. Several mechanisms contribute to acute kidney injury (AKI) in such patients, including renal vein compression with impairment of the venous drainage. Renal artery vasoconstriction induced by the neuro-hormonal systems worsens outcomes. The final result is progressive reduction in both glomerular perfusion and urine output. We present a case of 58-year-old man who underwent open cholecystectomy following a failed laparoscopic cholecystectomy attempt. Postoperatively, he developed AKI. This was attributed to pneumoperitoneum, an essential part of the laparoscopic procedure. This case illustrates that even when there are no obvious risk factors for postoperative complications, a high index of suspicion should be maintained for early recognition and treatment of complications from this surgery.
\end{abstract}

Keywords: Kidney; Laparoscopy; Cholecystectomy; Pneumoperitoneum; Complications

\section{Introduction}

The first successful laparoscopic cholecystectomy was performed in Europe in late 1980s [1]. Since then, this minimally invasive approach rapidly became the favored technique for the treatment of gallbladder disease in the United States. Apparent advantages such as decreased hospital length of stay, reduced cost, and increased patient satisfaction were the main reasons for the wide popularity of this technique.

Manuscript accepted for publication April 07, 2016

aDepartment of Internal Medicine, Seton Hall University of Health and Medical Science at St Francis Medical Center, Trenton, NJ, USA

${ }^{b}$ Corresponding Author: Ahmed Dirweesh, Department of Internal Medicine, Seton Hall University of Health and Medical Science at St Francis Medical Center, Trenton, NJ, USA. Email: adirweesh@stfrancismedical.org

doi: http://dx.doi.org/10.14740/jmc2483w
Currently, approximately 750,000 laparoscopic cholecystectomies are performed annually in the United States (accounting for roughly $90 \%$ of all cholecystectomies) with an overall serious complication rate that remains higher than that seen in open cholecystectomy, including bleeding, bile duct injury and bile leak, despite increasing familiarity with the procedure [2-4].

Pneumoperitoneum is used with laparoscopic surgery rise of the intra-abdominal pressure (IAP) and can lead to ischemic reperfusion kidney injury due to alterations in renal blood flow and reduction of the urinary output and creatinine clearance [5, 6]. Following desufflation of the abdomen, IAP and splanchnic blood flow normalize, signifying reperfusion.

\section{Case Report}

A 58-year-old Caucasian man with past medical history of essential hypertension, stable chronic obstructive airway disease, gastroesophageal reflux disease, osteoarthritis and attention deficit hyperactivity disorder came into the emergency room with right upper abdominal pain. The patient had a large fatty meal prior to pain onset. His pain was localized to RUQ, radiating to his back and right shoulder, 10/10 in intensity and had no relieving factors. Patient had 5 - 6 episodes of vomiting and reported having subjective fever associated with chills. Patient vitals were stable, afebrile, and cardiopulmonary examination was normal. His abdominal examination was positive for tenderness in RUQ and a positive Murphy's sign. His preoperative workup showed an elevated creatinine of $1.39 \mathrm{mg} / \mathrm{dL}$, and leukocytosis of 17.7 cells/ $\mu \mathrm{L}$. Radiological studies, abdominal ultrasound and HIDA scan, revealed cholelithiasis. No other abnormalities were noted. His mild acute kidney injury (AKI) on admission resolved with IV hydration and his creatinine prior to surgery came down to $0.86 \mathrm{mg} / \mathrm{dL}$. The patient underwent laparoscopic cholecystectomy which was then converted into open cholecystectomy due to procedure difficulties (multiple adhesions). The patient received adequate hydration intraoperatively and maintained an adequate urine output throughout the procedure. No complications occurred during the surgery and he tolerated the procedure well.

On the first postoperative day, the patient was started on clear liquids which were well tolerated, and IVFs were contin- 
ued. He was also started on vancomycin and zosyn. His postoperative workup revealed that he started developing AKI with creatinine of $2.7 \mathrm{mg} / \mathrm{dL}$ and BUN of $21 \mathrm{mg} / \mathrm{dL}$. The result of his blood work was normal. The patient remained asymptomatic. The nephrology team was consulted, vancomycin was held, and he continued to receive IV hydration. His creatinine worsened to $3.26 \mathrm{mg} / \mathrm{dL}$. The patient was asymptomatic during the postoperative period. He was fluid resuscitated, and over the next few days, his renal failure resolved. He was discharged home on day 7 and had no further deterioration in renal function.

\section{Discussion}

Laparoscopic surgery is rapidly replacing the open approach in many surgical fields, owing to its advantages including lesser pain and shorter postoperative hospital stay. Few cases of AKI following laparoscopic cholecystectomy are described in the literature and it has been reported mainly in patients with a history of chronic kidney disease [7].

Pneumoperitoneum, which is crucial to the laparoscopic technique, has a profound consequence on the patient's cardiopulmonary, gastrointestinal, and neuro-endocrine systems [3]. Normal IAP is $5-7 \mathrm{~mm} \mathrm{Hg}$ and is directly related to the body mass index. Intra-abdominal hypertension (IAH) is defined as a sustained IAP $\geq 12 \mathrm{~mm} \mathrm{Hg}$, whereas abdominal compartment syndrome is defined by a sustained abdominal pressure $>20$ $\mathrm{mm} \mathrm{Hg}$ that is associated with new organ dysfunction [8]. The kidney is the first organ usually affected by a rise in IAP [9].

Several mechanisms contribute to renal impairment in patients with IAH; renal vein compression with impairment of the venous drainage is the main one. Renal artery vasoconstriction induced by the neuro-hormonal systems worsens the outcome. The final result is progressive reduction in both glomerular perfusion and urine output [10]. These changes are often well tolerated in healthy patients [11]. Nevertheless, increased operative time as well as higher IAP increases the risk of developing such complications postoperatively even in normal individuals.

In the reported case, the occurrence of renal impairment was unanticipated as the patient did not have a prior history of renovascular disease. Vancomycin-induced nephrotoxicity was considered in the differential; however, levels were not sufficiently high to cause nephrotoxicity. His condition was related to pneumoperitoneum as there were no other intra- or postoperative explanations for his AKI.

\section{Conclusion}

This case illustrates that, even when there are no obvious risk factors for postoperative complications, a high index of suspicion should be maintained for early recognition and treatment of complications from this surgery. It is important to anticipate transient AKI in patients who have undergone laparoscopic abdominal surgeries and have longer intraoperative time [12]. Measures to correct causes of AKI and to prevent further dam- age should begin at the earliest signal of kidney injury. The essential goals of treatment are maintenance of volume homeostasis and correction of biochemical abnormalities. Prompt nephrology consultation should be considered, and with optimum medical care, most patients with normal preoperative renal functions are expected to achieve full recovery from the AKI.

\section{Grant Support}

None.

\section{Conflicts of Interest}

None.

\section{References}

1. Rosen M, Ponsky J. Minimally invasive surgery. Endoscopy. 2001;33:385-366.

2. Vollmer CM, Jr., Callery MP. Biliary injury following laparoscopic cholecystectomy: why still a problem? Gastroenterology. 2007;133(3):1039-1041.

3. Ben-David B, Croitoru M, Gaitini L. Acute renal failure following laparoscopic cholecystectomy: a case report. J Clin Anesth. 1999;11(6):486-489.

4. McLatchie G, Borley N, Chikwe J. Oxford handbook of clinical surgery. 3rd edn. Oxford: Oxford University Press, 2011.

5. Chiu AW, Chang LS, Birkett DH, Babayan RK. The impact of pneumoperitoneum, pneumoretroperitoneum, and gasless laparoscopy on the systemic and renal hemodynamics. J Am Coll Surg. 1995;181(5):397-406.

6. Carmona M, Lopes RI, Borba M, Omokawa M, Naufal R, Miyaji K, Matsumura N, et al. Comparison of the effects of carbon dioxide and helium pneumoperitoneum on renal function. J Endourol. 2008;22(5):1077-1082.

7. de Seigneux S, Klopfenstein CE, Iselin C, Martin PY. The risk of acute kidney injury following laparoscopic surgery in a chronic kidney disease patient. NDT Plus. 2011;4(5):339-341.

8. Malbrain ML, Cheatham ML, Kirkpatrick A, Sugrue M, Parr M, De Waele J, Balogh Z, et al. Results from the International Conference of Experts on Intra-abdominal Hypertension and Abdominal Compartment Syndrome. I. Definitions. Intensive Care Med. 2006;32(11):17221732.

9. Demyttenaere S, Feldman LS, Fried GM. Effect of pneumoperitoneum on renal perfusion and function: a systematic review. Surg Endosc. 2007;21(2):152-160.

10. Kirsch AJ, Hensle TW, Chang DT, Kayton ML, Olsson CA, Sawczuk IS. Renal effects of CO2 insufflation: oliguria and acute renal dysfunction in a rat pneumoperitoneum model. Urology. 1994;43(4):453-459.

11. Apostolou T, Sotsiou F, Dimitriades G, Nikolopoulou N, 
Chilal K, Balanika M, Yiannopoulos P, et al. Severe acute renal failure in a 19-year-old woman following laparoscopic cholecystectomy. Clin Nephrol. 2004;61(6):444-
447.

12. Briscoe $\mathrm{JH}$, Bahal $\mathrm{V}$. Acute renal failure following laparoscopic cholecystectomy. BMJ Case Rep. 2012;2012. 